\title{
SOME REMARKS NEAR-P-POLYAGROUPS AND POLYAGROUPS
}

\section{Janez Ušan and Mališa Žižović*}

\begin{abstract}
In this paper the Hosszú-Gluskin Theorem for near- $P$-polyagroups (polyagroups) is proved.
\end{abstract}

\section{Introduction}

1.1. Definition [1]: Let $n \geq 2$ and let $(Q, A)$ be an $n$-groupoid. We say that $(Q ; A)$ is a Dörnte $n$-group [briefly: $n$-group] iff is an $n$-semigroup and an n-guasigroup as well. (See, also [12].)

1.2. Definition (cf. [9],[10]): Let $k>1, s \geq 1, n=k \cdot s+1$ and let $(Q ; A)$ be an $n$-groupoid. Then: we say that $(Q ; A)$ is a polyagroup of the type $(s, n-1)$ iff the following statements hold:

$1^{\circ}$ For all $i, j \in\{1, \ldots, n\}(i<j)$ if $i \equiv j(\bmod s)$, then $\langle i, j\rangle$ - associative law holds in $(Q ; A)$; and

$2^{\circ}(Q ; A)$ is an $n$-quasigroup.

1.3. Definition[11]: Let $k>1, s \geq 1, n=k \cdot s+1$ and let $(Q ; A)$ be an $n$-groupoid. Then: we say that $(Q ; A)$ is a near-P-polyagroup [briefly: $N P$ - polyagroup $]$ of the type $(s, n-1)$ iff the following statements hold:

${ }^{\circ} 1$ For all $i, j \in\{1, \ldots, n\}(i<j)$ if $i, j \in\{t \cdot s+1 \mid t \in\{0,1, \ldots, k\}\}$, then the $\langle i, j\rangle-$ associative law holds in $(Q ; A)$; and

${ }^{\circ} 2$ For all $i \in\{t \cdot s+1 \mid t \in\{0,1, \ldots, k\}\}$, and for every $a_{1}^{n} \in Q$ there is exactly one $x_{i} \in Q$ such that the equality

holds. ${ }^{1)}$

$$
A\left(a_{1}^{i-1}, x_{i}, a_{i}^{n-1}\right)=a_{n}
$$

AMS (MOS) Subject Classification 1991. Primary: $20 \mathrm{~N} 15$.

Key words and phrases: $n$-groupoid, $n$-quasigroup, $n$-group, polyagroup, near- $P$ polyagroup.

* Research supported by Science Fund of Serbia under Grant 1457.

${ }^{1)}$ For $s=1(Q ; A)$ is a $(k+1)$-group, where $k+1 \geq 3 ; k>1$. 
1.4. Proposition: Every polyagroup of the type $(s, n-1)$ is an NPpolyagroup of the type $(s, n-1)$. [ By Def.1.2 and by Def. 1.3.]

\section{Auxiliary propositions}

2.1. Proposition [8]: Let $n \geq 2$ and let $(Q ; A)$ be an $n$-groupoid. Then, the following statements are equivalent: $(i)(Q ; A)$ is an n-group; (ii) there are mappings ${ }^{-1}$ and $\mathbf{e}$, respectively, of the sets $Q^{n-1}$ and $Q^{n-2}$ into the set $Q$ such that the following laws hold in the algebra $\left(Q ; A,{ }^{-1}, \mathbf{e}\right)$ lof the type $<n, n-1, n-2>$ ]

(a) $A\left(x_{1}^{n-2}, A\left(x_{n-1}^{2 n-2}\right), x_{2 n-1}\right)=A\left(x_{1}^{n-1}, A\left(x_{n}^{2 n-1}\right)\right)$,

(b) $A\left(\mathbf{e}\left(a_{1}^{n-2}\right), a_{1}^{n-2}, x\right)=x$ and

(c) $A\left(\left(a_{1}^{n-2}, a\right)^{-1}, a_{1}^{n-2}, a\right)=\mathbf{e}\left(a_{1}^{n-2}\right)$; and

(iii) there are mappings ${ }^{-1}$ and $\mathbf{e}$, respectively, of the sets $Q^{n-1}$ and $Q^{n-2}$ into the set $Q$ such that the following laws hold in the algebra $\left(Q ; A,{ }^{-1}, \mathbf{e}\right)$ lof the type $<n, n-1, n-2>$ ]

( $\bar{a}) A\left(A\left(x_{1}^{n}\right), x_{n+1}^{2 n-1}\right)=A\left(x_{1}, A\left(x_{2}^{n+1}\right), x_{n+2}^{2 n-1}\right)$,

(b) $A\left(x, a_{1}^{n-2}, \mathbf{e}\left(a_{1}^{n-2}\right)\right)=x$ and

(c) $A\left(a, a_{1}^{n-2},\left(a_{1}^{n-2}, a\right)^{-1}\right)=\mathbf{e}\left(a_{1}^{n-2}\right)$.

2.2. Remark: e is an $\{1, n\}$-neutral operation of $n$-groupoid $(Q ; A)$ iff algebra $(Q ; A, \mathbf{e})$ [of the type $\langle n, n-2\rangle$ ] satisfies the laws $(b)$ and $(\bar{b})$ from 2.1 [5]. Operation ${ }^{-1}$ from 2.1. [(c), $\left.(\bar{c})\right]$ is a generalization of the inverse operation in a group [6]. Cf. [12].

2.3. Definition[7]: We say that an algebra $(Q ; B, \varphi, b)$ [of the type $\langle 2,1,0\rangle$ is a Hosszú-Gluskin algebra of order $n(n \geq 3)$ [briefly: $n H G$-algebra] iff the following statements hold:

$(Q ; B)$ is a group;

$\varphi \in$ Aut $(Q ; B)$

$\varphi(b)=b ;$ and

For every $x \in Q, B\left(\varphi^{n-1}(x), b\right)=B(b, x)$.

2.4. Proposition (Hosszú-Gluskin Theorem [2,3])[7]: Let $(Q ; A)$ be an ngroup, e its $\{1, n\}$-neutral operation (cf. 2.2) and $n \geq 3$. Let also, $c_{1}^{n-2}$ be an arbitrary (fixed) sequence over a set $Q$, and let

$$
\begin{aligned}
& B(x, y) \stackrel{\text { def }}{=} A\left(x, c_{1}^{n-2}, y\right), \\
& \varphi(x) \stackrel{\text { def }}{=} A\left(\mathbf{e}\left(c_{1}^{n-2}\right), x, c_{1}^{n-2}\right) \text { and } \\
& b \stackrel{n \text { def }}{=} A\left(\frac{n}{\mathbf{e}\left(c_{1}^{n-2}\right) \mid}\right)
\end{aligned}
$$

for all $x, y \in Q$. Then, the following statements hold:

(1) $(Q ; B, \varphi, b)$ is an $n H G$-algebra; and 
(2) For every $x_{1}^{n} \in Q$ the equality holds. ${ }^{3)}$

$$
A\left(x_{1}^{n}\right)=\stackrel{n}{B}\left(x_{1}, \varphi\left(x_{2}\right), \ldots, \varphi^{n-1}\left(x_{n}\right), b\right)^{2)}
$$

2.5. Proposition [11]: Every $N P$-polyagroup has a $\{1, n\}$-neutral operation.

\section{Results}

3.1. Theorem: Let $k>1, s \geq 1, n=k \cdot s+1,(Q ; A)$ be an $N P$-polyagroup of the type $(s, n-1)$, e its $\{1, n\}$-neutral operation and $Y$ stands for sequence (1) $y_{1}^{s-1}, \ldots, y_{1}^{s-1} \in Q\left[=y_{1}^{s-1} \mid{ }_{j=1}^{k}\right]$ over $Q$. Also let $c_{1}^{k-1}$ be an arbitrary (fixed) sequence over a set $Q$. Further on, let

(1) $\mathbf{B}(Y, x, y) \stackrel{\text { def }}{=} A\left(x, y_{1}^{s-1}, c_{j} \mid \begin{array}{l}k-1 \\ j=1\end{array}, y_{1}^{(k)}, y\right)$

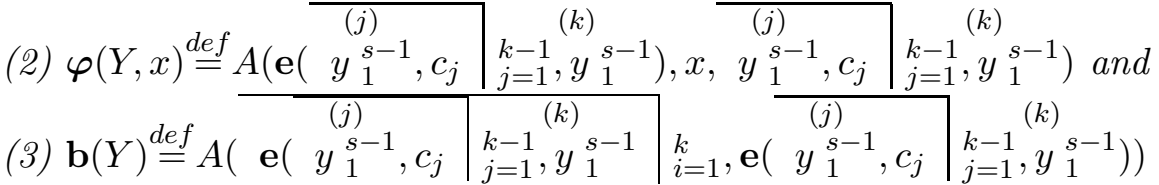

for all $x, y, y_{1}^{s-1}, \ldots, y_{1}^{s-1} \in Q$. Finally, let for all $x, y \in Q$

(1) $B_{Y}(x, y) \stackrel{\text { def }}{=} \mathbf{B}(Y, x, y)$,

(2) $\varphi_{Y}(x) \stackrel{\text { def }}{=} \varphi(Y, x)$ and

(3) $b_{Y} \stackrel{\operatorname{def}}{=} \mathbf{b}(Y)$,

where $Y$ is an arbitrary (fixed) sequence over $Q$. Then, the following statements hold:

(i) For all sequence $Y$ over $Q\left(Q ; B_{Y}, \varphi_{Y}, b_{Y}\right)$ is an $(k+1) H G$-algebra; and

(1) $\quad(k)$

(ii) For all $x_{1}^{k+1}, y_{1}^{s-1}, \ldots, y_{1}^{s-1} \in Q$ the following equality holds

$$
\begin{aligned}
& A\left(x_{j},\left.y_{1}^{s-1}\right|_{j=1} ^{k}, x_{k+1}\right)= \\
& \mathbf{B}\left(Y, x_{1}, Y, \varphi\left(Y, x_{2}\right), \ldots, Y, \varphi^{k}\left(Y, x_{k+1}\right), Y, \mathbf{b}(Y)\right),
\end{aligned}
$$

where $\varphi^{1} \stackrel{\text { def }}{=} \varphi$ and for all $x \in Q$, for all sequence $Y$ over $Q$ and for every $i \in N \varphi^{i+1}(Y, x) \stackrel{\text { def }}{=} \varphi\left(Y, \varphi^{i}(Y, x)\right)$.

2) $\stackrel{1}{B} \stackrel{\text { def }}{=} B$ and $\stackrel{t+1}{B}\left(x_{1}^{(t+1)(n-1)+1}\right) \stackrel{\text { def }}{=} B\left(\stackrel{t}{B}\left(x_{1}^{t(n-1)+1}\right), x_{t(n-1)+2}^{(t+1)(n-1)+1}\right), t \in N$; cf. [12], VI- 6 .

${ }^{3)}$ The formulation and the proof of the theorem follow the idea of E.I. Sokolov from [4]. See, also [12]; Chapter IV and Appendix 2. 
Proof. Firstly, we observe that under the assumptions the following statements hold

$1^{\circ}$ Let $Y$ be an arbitrary (fixed) sequence over a set $Q$. Also let

(4) $\mathbf{A}\left(x_{1}^{k+1}\right) \stackrel{\text { def }}{=} A\left(x_{j}, y_{1}^{s-1}{ }_{j=1}^{k}, x_{k+1}\right)$

for all $x_{1}^{k+1} \in Q$. Further on, let $c_{1}^{n-2}$ be an arbitrary (fixed) sequence over a set $Q$. Then $(Q ; \mathbf{A})$ is an $(k+1)$-group;

$2^{\circ}$ Let $(Q ; A)(k+1)$-group from $1^{\circ}$. Also let

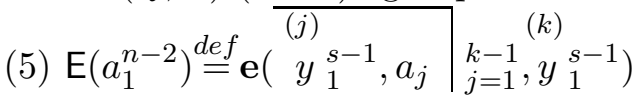

for all $a_{1}^{n-2} \in Q$. Then $\mathrm{E}$ is an $\{1, k+1\}$-neutral operation of the $(k+1)$-group $(Q ; \mathbf{A}) ;$ and

$3^{\circ}$ Let $(Q ; \mathbf{A})(k+1)$-group from $1^{\circ}$. Then:

$3_{a}^{\circ}\left(Q ; B_{Y}, \varphi_{Y}, b_{Y}\right)$ is an $(k+1) H G$-algebra; and

$3_{b}^{\circ} \mathbf{A}\left(x_{1}^{k+1}\right)={ }^{k+1} B_{Y}\left(x_{1}, \varphi_{Y}\left(x_{2}\right), \ldots, \varphi_{Y}^{k}\left(x_{k+1}\right), b_{Y}\right)$ for all $x_{1}^{k+1} \in Q$.

The proof of $1^{\circ}$ : Def. 1.1 and by Def. 1.3.

Sketch of the proof of $2^{\circ}$ :

$$
\begin{aligned}
& \mathbf{A}\left(\mathrm{E}\left(a_{1}^{n-2}\right),\right.\left.a_{1}^{n-2}, x\right) \stackrel{(5)}{=} \mathbf{A}\left(\mathbf { e } \left(y_{1}^{s-1}, a_{j}\right.\right. \\
&\left.\left.\stackrel{(j)}{k-1}, y_{1}^{s-1}\right), a_{1}^{n-2}, x\right) \\
& \\
& \stackrel{(4)}{=} A\left(\mathbf{e}\left(y_{1}^{s-1}, a_{j} l_{j=1}^{k-1}, y_{1}^{(k)}\right), y_{1}^{s-1}, a_{j}\right.\left.\begin{array}{l}
k-1 \\
j=1
\end{array}, y_{1}^{s-1}, x\right) \\
& \stackrel{2.5}{=} x .
\end{aligned}
$$

Whence, by Def. 1.1, Prop. 2.1 and by Rem. 2.2 , we obtain $2^{\circ}$.

Sketch of the proof of $3^{\circ}$ :

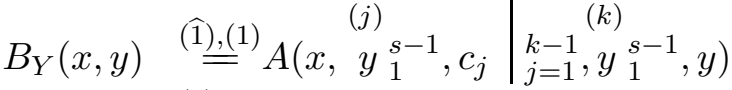

$$
\begin{aligned}
& \stackrel{(4)}{=} \mathbf{A}\left(x, c_{1}^{k-1}, y\right) \text {, }
\end{aligned}
$$

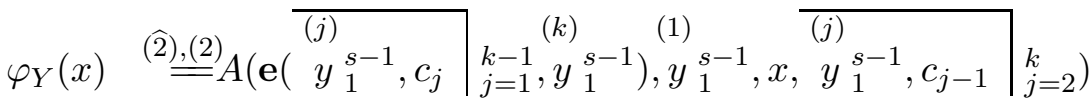

$$
\begin{aligned}
& \stackrel{(5)}{=} A\left(\mathrm{E}\left(c_{1}^{k-1}\right), y_{1}^{s-1}, x, y_{1}^{s-1}, c_{j-1} \mid \begin{array}{l}
k \\
j=2
\end{array}\right) \\
& \stackrel{(4)}{=} \mathbf{A}\left(\mathrm{E}\left(c_{1}^{k-1}\right), x, c_{1}^{k-1}\right) \text { and }
\end{aligned}
$$

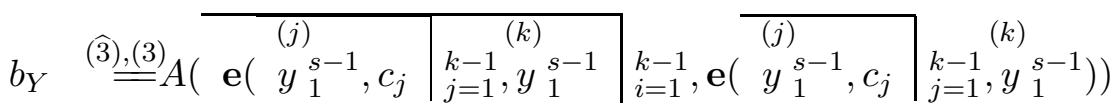

$$
\begin{aligned}
& \stackrel{(4),(5)}{=} \mathbf{A}\left(\frac{k+1}{\mathrm{E}\left(c_{1}^{k-1}\right) \mid}\right) \text {. }
\end{aligned}
$$


Whence, by Prop. 2.4, we obtain $3^{\circ}$.

In addition, by $3^{\circ}$, since $Y$ is an arbitrary sequence over $Q$, we conclude that the statement $(i)$ holds.

Finally, by $3^{\circ}\left[3_{b}^{\circ}\right],(\widehat{1})-(\widehat{3})$ and $(1)-(3)$, since $Y$ is an arbitrary sequence over $Q$, we obtain also $(i i)$.

By Th.3.1 and by Prop.1.4, we have:

3.2. Theorem: Let $k>1, s>1, n=k \cdot s+1,(Q ; A)$ be an polyagroup of the type $(s, n-1)$, e its $\{1, n\}$-neutral operation and $Y$ stands for sequence (1) $y_{1}^{s-1}, \ldots, y_{1}^{s-1}\left[=\left.y_{1}^{s-1}\right|_{j=1} ^{k}\right]$ over $Q$. Also let $c_{1}^{k-1}$ be an arbitrary (fixed) se-

quence over $Q$. Further on, let (1)-(3) from Th.3.1 for all $x, y, y_{1}^{s-1}, \ldots, y_{1}^{s-1} \in$ $Q$. Finally, let for all $x, y \in Q(\widehat{1})-(\widehat{3})$ form Th.3.1, where $Y$ is an arbitrary (fixed) sequence over $Q$. Then, the statements $(i)$ and (ii) from Th. 3.1 hold.

\section{References}

[1] W. Dörnte, Untersuchengen über einen verallgemeinerten Gruppenbegriff, Math. Z. 29(1928), 1-19.

[2] M. Hosszú, On the explicit form of $n$-group operations, Publ. math., Debrecen, 10, 1-4 (1963), 88-92.

[3] L.M. Gluskin, Position operatives, (Russian), Mat. sb., t. (68)(110), No. 3(1965), 444-472.

[4] E.I. Sokolov, On the Gluskin-Hosszú theorem for Dörnte n-groups, (Russian), Mat. Issled. 39(1976), 187-189.

[5] J. Ušan, Neutral operations of $n$-groupoids, (Russian), Rev. of Research, Fac. of Sci. Univ. of Novi Sad, Math. Ser. 18(1988) No. 2, 117-126.

[6] J. Ušan, A comment on n-groups, Rev. of Research, Fac. of. Sci. Univ. of Novi Sad, Math. Ser. 24(1994) No. 1, 281-288.

[7] J. Ušan, On Hosszú-Gluskin algebras corresponding to the same $n$-group, Rev. of Research, Fac. of Sci. Univ. of Novi Sad, Math. Ser. 25(1995), No.1, 101-119.

[8] J. Ušan, $n$-groups as variety of type $<n, n-1, n-2>$, in: Algebra and Model Theory, (A.G. Pinus and K.N. Ponomaryov, eds.) Novosibirsk 1997, 182-208.

[9] F.M. Sokhatsky, On the associativity of multiplace operations, Quasigroups and Related Systems 4(1997), 51-66.

[10] F.M. Sokhatsky and O. Yurevich, Invertible elements in associates and semigroups 2, Quasigroups and Related Systems 6(1999), 61-70.

[11] J. Ušan and R. Galić, On NP-polyagroups, Math. Communications, Vol.6(2001) No. 2, 153-159. 
[12] J. Ušan, n-groups in the light of the neutral operations, Math. Moravica special Vol. (2003), monograph.

Institute of Mathematics University of Novi Sad Trg D. Obradovića 421000 Novi Sad Serbia \& Montenegro Faculty of Tehnical Science University of Kragujevac Svetog Save 65, 32000 Čačak Serbia \& Montenegro 